\title{
Modeling the dual-function effects of hybrid- photopolymerization in Iodonium/Amine and two monomers system
}

\author{
Jui-Teng $\operatorname{Lin}^{1, *}$ \\ 1 New Vision Inc., 10F, No. 55, Sect.3, Xinbei Blvd, Xinzhuang, New Taipei City, Taiwan; \\ jtlin55@gmail.com \\ * Correspondences: jtlin55@gmail.com
}

\begin{abstract}
The synergetic features of a three-component photoinitiating systems $(\mathrm{A} / \mathrm{B} / \mathrm{C})$ based on the measured data and proposed mechanism of Liu et al are analyzed. The co-initiators/additives $B$ and $C$ have dual-functions of : (i) regeneration of photoinitiator A, and (ii) generation of extra radicals for enhanced conversion efficacy (CE), the synergic effects lead to higher $C E$ for both free radical polymerization (FRP) and cationic polymerization (CP). The CE of FRP has 3 terms due to the direct (tyep-I) coupling and two radicals. Therefore, it is always higher than that of $\mathrm{CP}$ having only one radical. The $\mathrm{CE}$ of $\mathrm{CP}$ has a transient state proportional to the effective absorption constnat (b), the light intensity (I) and initiator concentration $\left(\mathrm{A}_{0}\right)$, but a steady state given independent to the light intensity (I). For the CE of FRP, the contribution from radical $R$ could have two cases: (i) linear dependence on $\mathrm{T}^{\prime}=\mathrm{bIA}_{0}$, or (ii) nonlinear square root dependence $\mathrm{T}^{0.5}$. The nonlinear feature is due to the bimolecular termination term of $\mathrm{k}^{\prime} \mathrm{R}^{2}$. The key factors influencing the conversion efficacy are explored by analytic formulas. The synergetic effects lead to higher conversion of FRP and CP are consistent with the measured work. However, there are other theoretically predicted new features (findings) which are either not identified or explored experimentally including: (i) co-initiator [C] always enhances both FRP and CP conversions, whereas co-initiator [B] leads to more efficient FRP, but it also reduces $\mathrm{CP}$. The specific systems analyzed are: benzophenone derivatives (A) ethyl 4(dimethylamino)benzoate (B), and (4-tert-butylphenyl)iodonium hexafluorophosphate (C) under a UV (365 nm) LED irradiation; and two monomers of trimethylolpropane triacrylate (TMPTA, for FRP) and (3,4- epoxycyclohexane)methyl 3,4-epoxycyclohexylcarboxylate (EPOX, for CP).
\end{abstract}

Keywords: polymerization kinetics; photoinitiator; monomer conversion profile; synergetic effects.

\section{Introduction}

Comparing to the conventional thermal-initiated polymerization, there are several advantages for photopolymerization such as fast and controllable reaction rates, and spatial and temporal control over the formation of the material, without the need for high temperatures or harsh conditions [1]. Photopolymerizations using various light with wavelength in UV, visible and near IR have been studied for both industrial and medical applications. Variety of photoresponsive materials such as conjugated polymers have been reported for additive manufacturing (AM) and recently for 3D and $4 \mathrm{D}$ bioprinting [3,4]. For 3D photo printings, the key factors include polymerization depth, resolution precision and speed, in which the monomer conversion efficacy could be improved by various strategies. The reported conversion enhancing methods include the use of novel materials as enhancers or co initiators in both single and multiple components [5-8]. Two stage polymerization under two wavelengths to eliminate the oxygen inhibition effects was also reported experimentally [9-11]. Sequential network formation has also been achieved with many different types of 
polymerization methods, such as thiol-Michael/acrylate hybrid, epoxy/acrylate curable resins, thiolacrylate/thiol-acetoacetate thermosets, and thiol-ene/epoxy-based polymers [12-14].

UV light (at $365 \mathrm{~nm}$ ) have been commonly used in most type-I photoinitiators for the photopolymerization of (meth)acrylate monomers [1-3]. However, the UV wavelength suffers the disadvantages of being unsafe to skin and eyes, small penetration depth and larger light scattering in tissues [1]. Camphorquinone (CQ), due to its good visible absorption properties, is the most common type-II PI for the polymerization of (meth)acrylates under visible light $[15,16]$.

Various strategies to reduce oxygen inhibition in photoinduced polymerization have been proposed such as using co-initiators or addition of oxygen scavengers, and the thiol-ene and thiolacrylate-Michael systems which are insensitive to oxygen [8,9]. Dual-wavelength (red and UV) photopolymerization was also reported, in which pre-irradiation of the red light eliminated the oxygen inhibition effect and thus enhanced the conversion efficacy of the UV light [10].

The classical diaryliodonium salts, such as diaryliodonium, suffer low solubility in monomers and formation of side products due to the release of HF. To overcome this drawback, Kirschner et al. [15] recently reported a new counter anion-free and fluoride-free aryliodonium ylides (AY) to avoid the formation of HF and to enhance their solubility. They reported (CQ)/amine/AY as a new and efficient PI system for the polymerization of methacrylates under air and blue light $(477 \mathrm{~nm})$ irradiation for additional reactions and initiating radicals for improved conversion efficacy.

Various strategies for enhanced conversion were reported including the use of multiple light wavelength [9-11, 20-21], and co-initiators [12-14, 23].

Example of dual-wavelength (UV $365 \mathrm{~nm}$ and blue $470 \mathrm{~nm}$ ) system for enhanced conversion by reducing the oxygen inhibition was reported by de Beer et al $[8,9]$ for the photopolymerization of methacrylate formulated with camphorquinone (CQ) and ethyl 4-(dimethylamino)benzoate (EDAB), where CQ is the blue-light active initiator (A), butyl nitrite (BN) is the UV-activated initiator (B), and $\mathrm{EDAB}$ is a co-initiator (or donor $\mathrm{D}$ ). The photochemical decomposition of $\mathrm{BN}$ results in the formation of nitric oxide $(\mathrm{N})$, an efficient inhibitor of radical-mediated polymerizations, and alkoxide radical $(\mathrm{X})$ for extra polymerization initiation, besides the initiation radical $(\mathrm{R})$ generated from the blue-light

Example of 2-wavelength (red and UV) system (without the blue-light) for 3D printing was reported by Childress et al [10], in which a monomer of ethyl ether acrylate (DEGEEA) mixed by zinc 2,9,16,23-tetra-tert-butyl-29H,31H-phthalocyanine (ZnTTP) as an initiator under a UV-light, where ZnTTP/DEGEEA has distinct absorption peak at UV-365 nm and red-635 nm, respectively, and thus it can be independently excited by a UV and a red light, respectively. Lin et al [19] reported the theoretical modeling for the above described 2-wavelength system [13]. The novel strategy using 3wavelength of uv, blue and red lights was recently proposed by Lin et al [21] theoretically for future experimental studies.

Various reported enhancing strategies for photopolymerization including one component (or monomer) and one-wavelength [15-18,22], two-component and one wavelength 12,14], two wavelengths $[9-11,19,20)$ and three-wavelength [21]; and three-component, one-wavelength system $[13,23]$. We note that all these systems have been theoretically and experimentally studied, except the 3 -wavelength systems which was recently proposed theoretically by Lin et al [21]. The synergetic effects lead to higher monomer conversion can be achieved by co-initiators extra radicals and multiple wavelengths for reduced oxygen-inhibition.

This article will present, for the first time, the kinetics of the synergetic features of the 3-initiator, $[A],[B]$ and $[C]$, system based on the measured data and proposed mechanism of Liu et al [22], The co-initiator [B] and $[\mathrm{C}]$ have dual functions of: (i) regeneration of initiator $[A]$; and (ii) generation of extra radicals. The synergetic effects lead to higher conversion for FRP and CP. The key factors and rate constants influencing the conversion efficacy will be explored by analytic formulas of the conversion rate functions, derived from a kinetic model for a 3-initiator and 2-monomer system. While certain features predicted by this article are consistent with the measured data of Liu et al [22], some new theoretically predicted new finding are explored for future experimental studies. 


\section{Methods and Modeling Systems}

\subsection{Photochemical Kinetics}

As shown by Figure 1, a 3-initiator system (A/B/C) defined by the ground state of initiator[A], which is excited to its first-excited state $\mathrm{PI}^{*}$, and a triplet excited state $\mathrm{T}$ by a quantum yield (q). The triplet state $\mathrm{T}$ interacts with initiator $[\mathrm{A}]$ and $[\mathrm{B}]$ to produce radical $\mathrm{R}$; and interacts with co-initiator [C] to produce radical S'and S; in which the coupling of the radicals $\mathrm{R}^{\prime \prime}$ with [C] and $S^{\prime}$ with [B] lead to the regeneration of [A]. Radicals $R$ and $S$ lead to the free radical polymerization (FRP), and radical $S^{\prime}$ leads to cationic polymerization (CP), via monomers $M$ and $\mathrm{M}^{\prime}$. For system with [A] alone, $T, R^{\prime}$ and $R$ could be responsible for FRP, in general. In general, the terminations of our proposed scheme include the couplings of $R+R$ (bimolecule), $R+R^{\prime}$, and $R+S^{\prime}$, and $S+S$. I the two monomers system, $(T, R, S)+M$ (for FRP); $S^{\prime}+M^{\prime}$ (for $C P$ ). We will show later that Figure 1 is more general than the proposed Scheme of Liu et al [22].

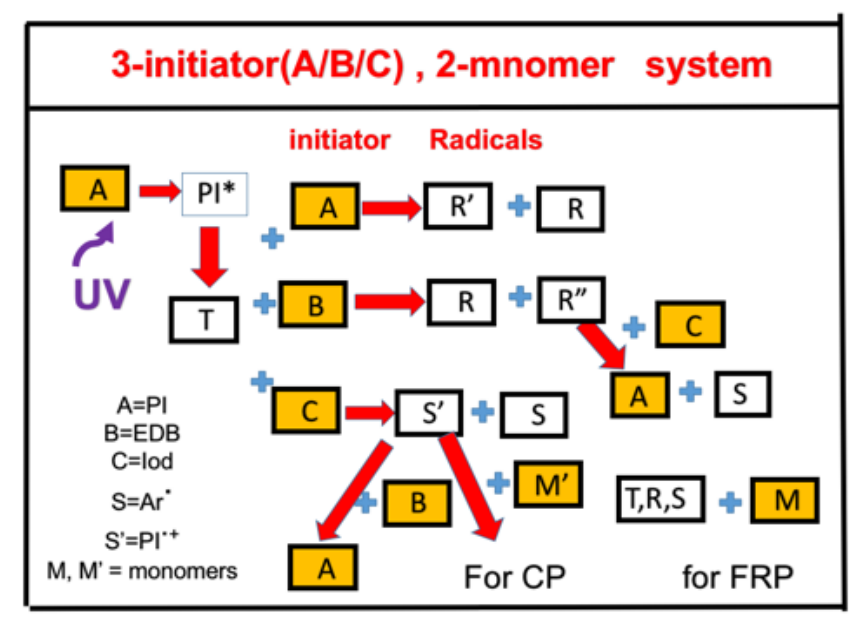

Figure 1. The schematics of a 3-initiator system, $(A / B / C)$, where $[A]$ is the ground state initiator, having a first excited state $\mathrm{PI}^{*}$, and a triplet state $\mathrm{T}$, which interacts with initiator $[\mathrm{A}]$ and $[\mathrm{B}]$ to produce radical $R$; and interacts with initiator $[C]$ to produce radical $S$, in which the coupling of the radical $\mathrm{R}^{\prime \prime}$ with $[\mathrm{C}]$ and $\mathrm{S}^{\prime}$ with $[\mathrm{B}]$ could lead to the regeneration of $[\mathrm{A}]$.

A specific system reported by Liu et al [22] is shown by the mechanism of Figure 1, where [A] is benzophenone (BP) photoinitiator, co-initiator [B] is ethyl 4-(dimethylamino)benzoate (EDB), and [C] is (4-tert-butylphenyl)iodonium hexafluorophosphate (Iod). Under a UV (365 nm) LED irradiation, [A] transforms from ground state (PI) to excited triple state 1,3PI (shown by r1), which couples with PI to produce radicals PI-Ho (or R) and PI(-H)o (or $\mathrm{R}^{\prime}$ ) (shown by $\mathrm{r} 2$ ). In the presence of EDB, extra radical $\mathrm{EDB}(-\mathrm{H})^{\circ}$ (or $\mathrm{R}$ ) and $\mathrm{PI}-\mathrm{H}^{\circ}$ (or $\mathrm{R}^{\prime \prime}$ ) are produced (shown by $\mathrm{r} 3$ ). $\mathrm{T}$ could couple with [C] to produce aryl radical $\mathrm{Ar}^{\circ}$ and $\mathrm{PI}^{\circ+}(\mathrm{r} 4)$, where radicals $\mathrm{Ar}^{\circ}$ (or S) and $\mathrm{EDB}(-\mathrm{H})^{\circ}$ (or R) lead to FRP and $\mathrm{PI}^{\mathrm{o}^{+}}$(or $\mathrm{S}^{\prime}$ ) leads to CP. Furthermore, PI-Ho (or R") could couple with Iod (or C) to produce extra radical Aro for FRP (r5) and also regenerate PI (or [A]). Radical $\mathrm{PI}^{\mathrm{o}+}$ (or $\mathrm{S}^{\prime}$ ) could couple with EDB to regenerate PI (or [A]), shown by r6. Associated with the photolysis of BPC1/Iod and BPC1/EDB/Iod, the photoredox catalytic cycle was proposed in three-component PI/EDB/Iod system (shown by r5 and r6). The regeneration of PI speeds up the photopolymerization and slows down the consumption of PI in the photolysis experiments. Trimethylolpropane triacrylate (TMPTA) and (3,4epoxycyclohexane)methyl 3,4-epoxycyclohexylcarboxylate (EPOX) were used as benchmark monomers for FRP and $\mathrm{CP}$, respectively.

However, we note that our Figure 1 is more general than the following Scheme of Liu et al [22] which ignored the termination scheme due to the couplings of $R+R$ (bimolecular), $R+S$, and $R+S$ '. Furthermore, the measured data of Liu et al [22] for the case of CP was limited to two initiators of [A] and $[\mathrm{C}]$, although 3-initiator systems of $[\mathrm{A}] /[\mathrm{B}] /[\mathrm{C}]$ was studied in FRP. Our modeled system of Figure 
1 and the associated kinetic equations to be shown later include 3-initiator for both FRP and CP. The mechanism of a 3-initiator and 2-monomer, $\mathrm{A} / \mathrm{B} / \mathrm{C} / \mathrm{M} / \mathrm{M}^{\prime}$ system proposed by Liu et al [22] is shown in the following schematic equations of (r1) to (r6).

$$
\begin{aligned}
& \mathrm{PI} \stackrel{\mathrm{h} v}{\longrightarrow} 1,3 \mathrm{PI} \\
& { }^{1,3} \mathrm{PI}+\mathrm{PI} \longrightarrow \mathrm{PI}^{\bullet-}+\mathrm{PI}^{\bullet+} \longrightarrow \mathrm{PI}-\mathrm{H}^{\bullet}+\mathrm{PI}_{(-\mathrm{H})} \\
& { }^{1,3} \mathrm{PI}+\mathrm{EDB} \longrightarrow \mathrm{PI}^{\bullet-}+\mathrm{EDB}^{\bullet+} \longrightarrow \mathrm{PI}-\mathrm{H}^{\bullet}+\mathrm{EDB}_{(-\mathrm{H})} \\
& { }^{1,3} \mathrm{PI}+\mathrm{Ar}_{2} \mathrm{I}^{+} \longrightarrow \mathrm{PI}^{\bullet+}+\mathrm{Ar}_{2} \mathrm{I}^{\bullet} \longrightarrow \mathrm{PI}^{\bullet+}+\mathrm{Ar}^{\bullet}+\mathrm{ArI} \\
& \mathrm{PI}-\mathrm{H}^{\bullet}+\mathrm{Ar}_{2} \mathrm{I}^{+} \longrightarrow \mathrm{PI}+\mathrm{Ar}{ }^{\bullet} \mathrm{ArI}+\mathrm{H}^{+} \\
& \mathrm{PI}^{\bullet+}+\mathrm{EDB} \longrightarrow \mathrm{PI}+\mathrm{EDB}^{\bullet+}
\end{aligned}
$$

Scheme 1. The proposed chemical reactions of a PI/EDB/Amine for a hybrid FRP and CP system with two monomers of TMPTA and EPOX [22].

Based on the above schematic equations, the initiator concentration, $[A],[B]$ and $[C]$, and the monomer $\mathrm{M}$ (for FRP) and and $\mathrm{M}^{\prime}$ (for $\mathrm{CP}$ ) are given by: (detailed derivatios are shown in Apendix)

$\frac{d M}{d t}=-\left(k T+K S+k^{\prime} R\right) M$

$\frac{d M^{\prime}}{d t}=-K^{\prime} S^{\prime} M^{\prime}$

Eq. (1) shows that FRP is due to 3 conributions: from the direct (type-I) coupling kTM term; the radical $S$ and radical $R$, whereas $C P$ is only from the catonic radical $S$ ', shown in Eq. (2). Numerical simulation is in general required for the solution of $M$ and $\mathrm{M}^{\prime}$, due to the inter-coupling of the radicals $\left(\mathrm{R}, \mathrm{S}, \mathrm{S}^{\prime}\right)$ and additives, $[\mathrm{A}],[\mathrm{B}]$ and $[[\mathrm{C}]$. We will focus on the synergis effcets based on analytic formulas, derived in the Appendix.

The steady state radical R, as given by the Appendix, Eq. (A11), is given by the solution of

$k^{\prime} R^{2}+G R-P=0$

where $\mathrm{G}=\mathrm{k}^{\prime \prime} \mathrm{S}+\mathrm{k}^{\prime} \mathrm{M}$, and $\mathrm{P}=\left(\mathrm{k}_{1}[\mathrm{~A}]+\mathrm{k}_{2}[\mathrm{~B}]\right)$, with $\mathrm{T}=\mathrm{bIg}[\mathrm{A}], \mathrm{b}$ is an effective coupling constnat given by $\mathrm{b}=83.6 a^{\prime} w q$, where $w$ is the UV light wavelength (in $\mathrm{cm}$ ) and $q$ is the triplet state T quantum yield; $a^{\prime}$ is the mole absorption coefficient, in $(1 / \mathrm{mM} / \%)$ and $\mathrm{I}(\mathrm{z}, \mathrm{t})$ is the light intensity.

Solving for R, we obtain

$R=\left(\frac{1}{2 k^{\prime}}\right)\left(-G+\sqrt{G^{2}+4 k^{\prime} H}\right)$

Analytic formulas of $\mathrm{R}$ is available under two special cases.

Case (i) for unimolecular termination dominant, or $\mathrm{G}>>\mathrm{k}$ ' $\mathrm{P}$, we obtain $\mathrm{R}=\mathrm{P} / \mathrm{G}$, which is a linear increasing function of $\left(\mathrm{k}_{1}[\mathrm{~A}]+\mathrm{k}_{2}[\mathrm{~B}]\right) \mathrm{T} /\left(\mathrm{k}^{\prime \prime} \mathrm{S}+\mathrm{k}^{\prime} \mathrm{M}\right)$. 
Case (ii) for bimolecular termination dominant, with $P \gg>G R$, we obtain, $R=\left[P / k^{\prime}\right]^{0.5}$. a nonlinear function of a square root function, $\left[\left(\mathrm{k}_{1}[\mathrm{~A}]+\mathrm{k}_{2}[\mathrm{~B}]\right) \mathrm{T} / \mathrm{k}^{\prime}\right]^{0.5}$.

\section{3.. Results and discussion}

\subsection{Formulas for conversion efficacy (CE)}

The $\mathrm{CE}$ of $\mathrm{CP}$ defined by $\mathrm{CE}^{\prime}=1-\mathrm{M}^{\prime} / \mathrm{Mo}^{\prime}$, which is the time integral of Eq. (2), we obtain , from Eq. (A13) of Appendix,

$$
\mathrm{CE}^{\prime}=\left[1-\mathrm{k}_{7} \mathrm{~B}_{0} /\left(\mathrm{K}^{\prime} \mathrm{Mo}^{\prime}\right)\right] \mathrm{K}^{\prime} \mathrm{D}\left(\mathrm{bIC}_{0} \mathrm{~A}_{0}\right) \mathrm{H}(\mathrm{t}),
$$

with $H(t)=\left[1-\exp \left(-d^{\prime} t\right)\right] / d^{\prime}$, with a revised $d^{\prime}=k_{3} T^{\prime} /\left(k M_{0}\right)+X^{\prime}$, for a non-constant $[A]=A 0 \exp \left(-X^{\prime} t\right)$. It shows that, in the presence of $[B]$, the $C P$ is reduced by a reduction factor of $F^{\prime}=\left[1-k_{7} B_{0} /\left(K^{\prime} \mathrm{Mo}^{\prime}\right)\right]$. The $C E^{\prime}$ has a transient state proportional to $\mathrm{bIt}\left(\mathrm{C}_{0} \mathrm{~A}_{0}\right)$, but a steady state given by $\mathrm{TE} \mathrm{E}^{\prime}=\mathrm{K} \mathrm{D}^{\prime}\left(\mathrm{bIC}_{0} \mathrm{~A}_{0}\right) / \mathrm{d}^{\prime}=$ $\mathrm{K}^{\prime} \mathrm{D}\left(\mathrm{C}_{0} \mathrm{~A}_{0}\right)\left[\mathrm{k}_{3} /\left(\mathrm{kM} \mathrm{M}_{0}\right)\right]$, which is independent to the light intensity (I).

The CE of FRP defined by TE=1-M/Mo, the solution of Eq. (1), which is much more complex and given by, from Eq. (A14) of Appendix, including the revised factor for $\mathrm{T}^{\prime}$

$$
\mathrm{CE}=\left(\mathrm{T}^{\prime \prime} / \mathrm{M}_{0}\right) \mathrm{t}+\mathrm{KD} \mathrm{H}^{\prime}(\mathrm{t})+\mathrm{KD} \mathrm{H}^{\prime} \mathrm{H}^{\prime}(\mathrm{t})+\mathrm{P}^{\prime} \mathrm{H}^{\prime \prime}(\mathrm{t}) \text {, }
$$

where a revised $T^{\prime \prime}=\left(b_{I A}\right)\left[1-\exp \left(-X^{\prime} t\right)\right] / X^{\prime}$. The above CE has 3 terms: (i) from the direct couplig of $T$ and $\mathrm{M}$; (ii) coupling of radical $\mathrm{S}$ and $\mathrm{M}$, and (iii) coupling of radical $\mathrm{S}$ and $\mathrm{M}$, the terms of $\mathrm{KD}^{\prime} \mathrm{H}^{\prime}$ and KD"' $\mathrm{H}$ "; and (iii) the last term for coupling of radical $\mathrm{R}$ and $\mathrm{M}$, given by $\mathrm{P}^{\prime} \mathrm{H}^{\prime \prime}$. For case (i) linear case, we obtain $\mathrm{H}^{\prime \prime}(\mathrm{t})=\mathrm{Pt}+\mathrm{P}^{\prime} \mathrm{H}^{\prime \prime}(\mathrm{t})$, with $\mathrm{H}^{\prime \prime}=\left[1-\exp \left(\mathrm{d}^{\prime \prime} \mathrm{t}\right)\right] / \mathrm{d}$ ", with $\mathrm{P}=\mathrm{k}_{1}\left(\mathrm{~A}_{0}{ }^{2} / \mathrm{M}_{0}\right) \mathrm{T}^{\prime}, \mathrm{P}^{\prime}=\mathrm{k}_{2}\left(\mathrm{~B}_{0} / \mathrm{M}_{0}\right) \mathrm{T}^{\prime}$, and $\mathrm{d}^{\prime \prime}=\left[\mathrm{k}_{2} \mathrm{~T}^{\prime} /\left(\mathrm{kM}_{0}\right)+0.5 \mathrm{k}_{7} \mathrm{k}_{3} \mathrm{~T}^{\prime} \mathrm{t}\left[\mathrm{C}_{0} /\left(\mathrm{kM}_{0} \mathrm{~K}^{\prime} \mathrm{M}^{\prime} \mathrm{o}\right)\right] \mathrm{t}\right.$. For case (ii) nonlinear square root case, $k^{\prime} R=\left[k^{\prime}\left(k_{1}[A]+k_{2}[B]\right) T\right]^{0.5}$, we obtain $H^{\prime \prime}(t)=V t+V^{\prime} H^{\prime \prime}(t)$, with $V=\left(k_{1}\left(A_{0} M_{0}\right) T^{\prime}\right)^{0.5}, V^{\prime}=\left(k_{2}\left(B_{0} M_{0}\right) T^{\prime}\right)^{0.5}$.

\subsection{Synergetic effects}

As shown by Figure 1, and Eqs. (1) to (6), the following synergetic features of the 3-initiator system $\mathrm{A} / \mathrm{B} / \mathrm{C}$ are summarized as follows.

(a) Co-initiator [B] has multiple functions of : (i) regeneration of initiator [A] leading to higher FRP conversion; (ii) producing of extra radical R and radical R"; and (iii) coupling of R" with [C] leads to radical $S$, in which both $R$ and $S$ lead to FRP.

(b) Similarly, co-initiator [C] has functions of : (i) regeneration of initiator [A] via the coupling of $\mathrm{S}^{\prime}$ and [B], leading to higher FRP conversion; (ii) generation of cationic radical $\mathrm{S}^{\prime}$ for $\mathrm{CP}$ conversion; and (iii) enhancing FRP by producing radical S (as shown by Figure 1).

(c) As shown by Eq. (5) and (6) that CE of FRP has 3 terms, kTM, KSM and k'R. Therefore it is always higher than that of $\mathrm{CP}$, only one term of $\mathrm{K}^{\prime} \mathrm{S}^{\prime} \mathrm{M}$ '.

(d) The presence of [C] alwyas leads to higher FRP via the extra radical S, and the regeneration of [A]. However, the presence of $[B]$ consumes radical $S^{\prime}$, and hence reduces the FRP. Therefore the net enhanced effects of $[C]$ on FRP is governed by the relative strength of regeneration of $[A]$ and the reduction effect, as shown by our formula in Eq. (5) with a reduction factor $F^{\prime}=\left[1-\mathrm{k}_{7} \mathrm{~B}_{0} /\left(\mathrm{K}^{\prime} \mathrm{M}^{\prime}\right)\right]$. These non-common features are not explored by Liu et al [22], and could not be easily predicted without the detailed mathematically efforts.

(e) From Eq. (5), the $\mathrm{CE}$ of $\mathrm{CP}$ has a transient state proportional to bIt $\left(\mathrm{C}_{0} \mathrm{~A}_{0}\right)$, but a steady state given independent to the light intensity (I). 
(f) From Eq. (6), the CE of FRP, the contribution from radical R could have two cases: (i) linear case of $\mathrm{R}$ on $\mathrm{T}^{\prime}=\mathrm{bIA}$, or (ii) nonlinear square root dependence $\left.\mathrm{T}\right]^{0.5}$. However, the contribution from radical $\mathrm{S}$ has linear dependence of $\mathrm{T}^{\prime}=\mathrm{bIA} 0$, The nonlinear feature is due to the bimolecular termination included in the coupling term of $\mathrm{k}^{\prime} \mathrm{R}^{2}$ in Eq. (A5).

(g) For a very weak type-I coupling (or $\mathrm{k}_{1}[\mathrm{~A}]<<1 / \mathrm{g}$, or type-II dominant, $\mathrm{d}[\mathrm{A}] / \mathrm{dt}=0$, which defines a perfect regeneration, or $[A]=A_{0}$ is a constant. In non-perfect regeneration, $[A]=A_{0} \exp (-X) t$, and the depeltion of $[\mathrm{A}]$ leads to a lower conversion, and a time-dependent light intensity deviating from the Ber Lambert law.

Above theoretically predicted new findings are not explored in the measured work of Liu et al [22].

Figure 2 shows the results based on Eq. (5) for CP conversion re-expressed as $C E^{\prime}=Q\left[1-\exp \left(-\mathrm{d}^{\prime} \mathrm{t}\right)\right]$, with $\mathrm{Q}=\left[1-\mathrm{k}_{7} \mathrm{~B}_{0} /\left(\mathrm{K}^{\prime} \mathrm{Mo}^{\prime}\right)\right] \mathrm{K}^{\prime} \mathrm{D}\left(\mathrm{bIC}_{0} \mathrm{~A}_{0}\right)$, for various values of $\mathrm{Q}$ and the initiator depeltion rate $\mathrm{d}^{\prime}$. We note that larger $Q$ achieves the higher steady-state profile, as shown by Figure 2(A). Furthermore, the profile rising rates are given by $\mathrm{d}$ (proportional to $\mathrm{bIA}_{0}$ ). Higher light intensity $(\mathrm{I})$, larger initiator concentration, or stronger absorption (b) leads to a faster depeltion of $[\mathrm{A}]$ and hence faster rising of the conversion profile, shown by Figure 2(B). We note that for the same $Q$ value, the CP conversion profiles having different d'-values (or bIg) reach the same steady state. However, we have previously reported different features of FRP [24] that larger d' value reaches a lower steady state conversion (not shown in this article), which is fundamentally different for FRP and CP profiles. Above theoretically predicted new findings are not explored in the measured work of Liu et al [22].
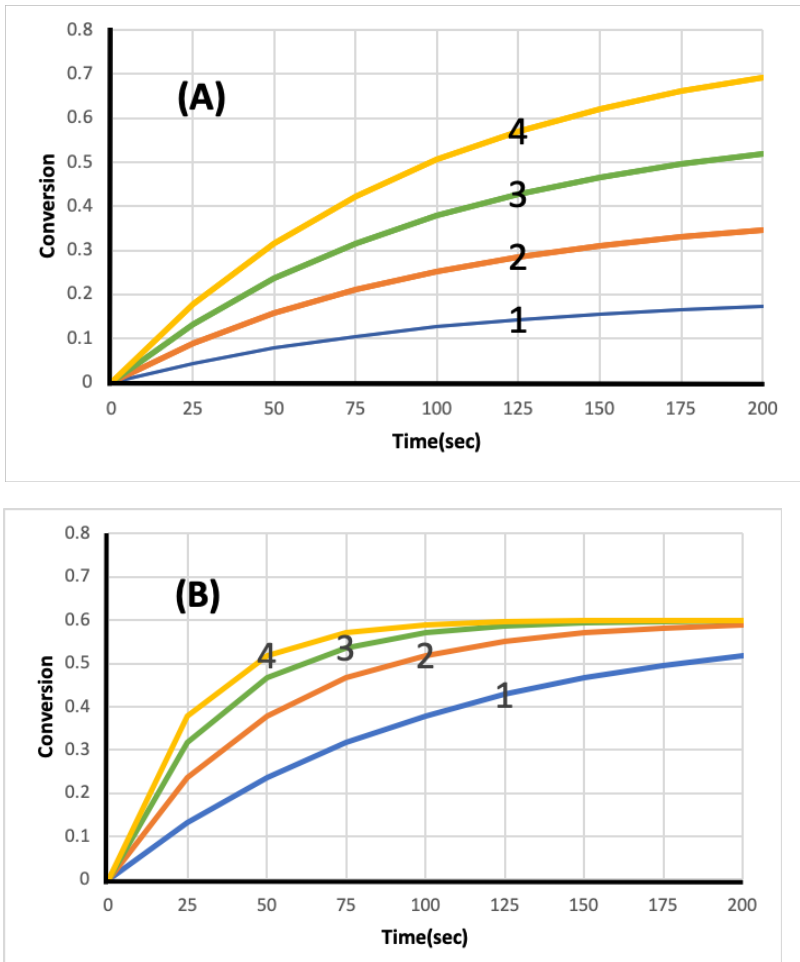

Figure 2. The calculated $C P$ conversion profiles for various $Q$ and $d$ ' values: $(A)$ for fixed $d '=0.01$, and $Q=(0.2$, $0.4,0.6,0.8)$, for Curves 1,2,3 and 4; and (B) for fixed $\mathrm{Q}=0.6$, and $\mathrm{d}^{\prime}=(0.01,0.02,0.03,0.04)$ for Curves $1,2,3$, and 4 , respectively.

\subsection{Analysis of measured results}


Besides the synergetic effects described in last section, our formulas shown by Eq. (18) to (23), may be used to analyze the measured results of Liu et al [22] as follows:

Fig. 3 of Liu et al [22] for cationic polymerization of EPOX showed that BPC1/Iod system has the highest conversion (44\%) due to its highest light absorption. The role of light absorption was shown by Eq. (A9) and (A10), in which the conversion rate is an increasing function of $b$, defined by $b=83.6 a^{\prime} w q$, where $a^{\prime}$ is the molar extinction coefficient, $w$ is the UV light wavelength and $q$ is the triplet state quantum yield. BPC1-BPC4 have higher molar extinction coefficients, therefore the CP conversion rate of EPOX for BPC1-BPC4/Iod systems are faster than C5-C8/Iod systems for the same initial concentration of PI and Iod. Fig. 3 of Liu et al [22] may be compared with our Figure 2(A), where higher $Q$ value leads to larger steady-state conversion, as also shown by Eq. (5) and (6).

Fig. $4 \mathrm{~b}$ of Liu et al [22] showed that higher FRP conversion of TMPTA in the presence of PI/EDB systems comparing to that of PI alone, shown by Fig. 4a. This enhanced polymerization is due to the increase of the conversion rate as shown by the additional term $\mathrm{k}_{2}[\mathrm{~B}]$ of Eq. (20). EDB (or co initiator [B]) as $\mathrm{H}$ donor has an effective interaction with PI to generate radicals which promote the free radical polymerization, so the PI/EDB systems have better polymerization performance than PI alone systems. Fig. $4 \mathrm{~d}$ of Liu et al [22] shown that FRP conversion of TMPTA initiated by PI/EDB/Iod (or $\mathrm{A} / \mathrm{B} / \mathrm{C}$ ) systems are better than PI/EDB (or $\mathrm{A} / \mathrm{B}$ ) and PI/Iod (or $\mathrm{A} / \mathrm{C}$ ) systems. This can be easily realized by our Eq. (1), that the conversion rate is an increasing function of $k T+K S+k ' R$. That is, $3-$ initiator system is more efficient than that of two (with $[B]$ or $[C]=0$ ) and one initiator (with $[B]=[C]=0$ ) systems. In PI/EDB/Iod system, the triple state (T) reacts with PI, EDB and Iod at the same time. There is a photoredox catalytic cycle in 3-initiator system and the regeneration of PI speeds up the polymerization, in additional to the free radicals (R,S and $\mathrm{S}^{\prime}$ ). Therefore, PI/EDB/Iod systems have better polymerization performances than PI/EDB and PI/Iod systems.

Fig. 5 of Liu et al [22] showed that the consumption rate of BPC1 in BPC1/TEOA/Iod system was slower than BPC1/Iod system. The photoredox catalytic cycle in the three-component system could regenerate BPC1, as shown by Eq. (1) and (8). Fig. 6 and S1 of Liu et al showed that the consumption rates of BPC1-BPC4 were faster than C5 and C7 due to the presence of the benzophenone moiety in BPC1-BPC4 structures which promoted the reaction between PI and amine. The photolysis demonstrated the benzophenone-carbazole PIs had high reactivity. Higher reactivity of co-initiator benzophenone leads to higher conversion, as shown by our Eq. (5) and (6).

Fig. 7 of Liu et al [22] showed the fluorescent properties as the evidence of the interaction capacity of PIs with additives in the excited singlet state. The role of triple-state quantum yield (q) was also shown by the $\mathrm{b}$ factor of Eq. (20) and (21). The fluorescence experiments demonstrated that benzophenone-carbazole PIs could be quenched easily by additives. The high electron transfer quantum yields show that electron transfer occurs effectively between benzophenone-carbazole PIs and EDB/Iod and therefore, lead to high polymerization conversions. These features are also predicted by our Eq. (5) and (6).

\section{Conclusion}

This article present, for the first time, the kinetics of the synergetic features of a 3-initiator, [A], $[B]$ and $[C]$, system based on the measured data and proposed mechanism of Liu et al [22]. The coinitiator [B] and [C] have dual functions of: (i) regeneration of initiator [A]; and (ii) generation of extra radicals. The synergetic effects lead to higher conversion for FRP and CP. These features are also shown by the measured work of Liu et al [22]. However, there are other theoretically predicted new features (findings) of this article, which are either not identified or explored by Liu et al [22], including:

(i) Co-initiator [C] always enhances both FRP and CP conversions, whereas co-initiator [B] leads to more efficient FRP, but it also consumes $[C]$ and thus reduces $C P$. 
(ii) The contribution of radical $\mathrm{R}$ for FRP could have two cases: (i) linear case of $\mathrm{R}$ on $\mathrm{T}^{\prime}=\mathrm{bIA}$, or (ii) nonlinear square root dependence $\mathrm{T}^{0.5}$. However, the contribution from radical $\mathrm{S}$ has linear dependence of $T^{\prime}=b I A$, The nonlinear feature is due to the bimolecular termination of radical $R$.

(iii) The steady state conversion profile of $\mathrm{CP}$, as shown by Figure 2 (B), reaches a constant and is independent to the light intensity, whereas higher light intensity reaches a lower steady state value for the profile of FRP.

Acknowledgments: JTL thanks the internal grant of New Vision Inc. and the computer drawing by Jonathan Y.K. Hu (Kaohsiung American School, Taiwan).

Conflicts of Interest: Jui-Teng Lin is the CEO of New Vision Inc.

\section{APPENDIX}

\section{Derivation of kinetic equations}

The kinetic equations for our previous 2-initiator and 1-monomer system [24-26] are revised for the 3initiator and 2-monomer system, A/B/C/M/M' , based on the schematic mechanisms of Eqs. (r1) to (r6), as follows. Also refer to the definitions shown in Figire 1.

$$
\begin{aligned}
& \frac{\partial[A]}{\partial t}=-b I(z, t)[A]+R G E \\
& \frac{\partial[B]}{\partial t}=-\left(k_{2} T+k_{7} S^{\prime}\right)[B] \\
& \frac{\partial[C]}{\partial t}=-\left(k_{3} T+k_{6} R^{\prime \prime}\right)[C] \\
& \frac{\partial T}{\partial t}=b I(z, t)[A]-\left(k_{5}+k_{1}[A]+k_{2}[B]+k_{3}[C]+k M\right) T \\
& \frac{\partial R}{\partial t}=\left(k_{1}[A]+k_{2}[B]\right) T-\left(k R+k^{\prime \prime} S+k^{\prime} M\right) R \\
& \frac{\partial R^{\prime \prime}}{\partial t}=k_{2}[B] T-k_{6} R^{\prime \prime}[C] \\
& \frac{\partial S}{\partial t}=k_{3} T[C]+k_{6} R^{\prime \prime}[C]-K S M-k^{\prime \prime} R S \\
& \frac{\partial S^{\prime}}{\partial t}=k_{3} T[C]-k_{7}[B] S^{\prime}-K^{\prime} S^{\prime} M^{\prime}
\end{aligned}
$$

where $R G E=k_{5} T+k_{6} R^{\prime \prime}[C]+k_{7} S^{\prime}[B]+k M$, is the regeneration term of of $[A] . b=83.6 a^{\prime} w q$, where $w$ is the UV light wavelength (in $\mathrm{cm}$ ) and $q$ is the triplet state T quantum yield; $a^{\prime}$ is the mole absorption coefficient, in $(1 / \mathrm{mM} / \%)$ and $\mathrm{I}(\mathrm{z}, \mathrm{t})$ is the light intensity, in $\mathrm{mW} / \mathrm{cm}^{2}$. All the rate constants are defined previously and they are related by the coupling terms. For examples, $k_{j}(w i t h j=1,2,3$ ) are for the couplings of $\mathrm{T}$ and $[\mathrm{A}],[\mathrm{B}]$, and $[\mathrm{C}]$, respectively; $\mathrm{k}_{6}$ and $\mathrm{k}_{7}$ are for the couplings of $\mathrm{R}$ and $[\mathrm{C}]$, and $\mathrm{S}^{\prime}$ and $[\mathrm{B}]$, respectively; and $\mathrm{k}_{\mathrm{T}}$ is the bimolecular termination rate of $\mathrm{S}$. The coupling among $\mathrm{T}, \mathrm{R}, \mathrm{S}$, and $\mathrm{S}$ and $\mathrm{M}\left(\mathrm{M}^{\prime}\right)$ for polymerization are given by $\mathrm{k}_{8}, \mathrm{k}_{89}, \mathrm{~K}$ and $\mathrm{K}^{\prime}$, respectively. 
The monomers conversions for FRP and CP given by M and M', resepctively, may be obtained by the following equations [23]

$$
\begin{aligned}
& \frac{d M}{d t}=-\left(k T+K S+k^{\prime} R\right) M \\
& \frac{d M^{\prime}}{d t}=-K^{\prime} S^{\prime} M^{\prime}
\end{aligned}
$$

which are governed by the interaction of $(T, R, S)$ and $M$; and $S^{\prime}$ and $M^{\prime}$, respectively.

We note that Eq. (1) to (10) are constructed for the specific system of Liu et al [22], in which the following couplings (or effects) are ignore: oxygen inhibition, couplings of $S$ and $S^{\prime}, R$ and $S, R$ and $[\mathrm{B}], \mathrm{R}$ and $[\mathrm{A}], \mathrm{S}^{\prime}$ and $[\mathrm{A}]$; and the direct coupling of initiators, $[\mathrm{A}],[\mathrm{B}],[\mathrm{C}]$ and the monomers, $\mathrm{M}$ and $\mathrm{M}^{\prime}$ (type-I processes). For [A] alone, we assume the FRP is mainly due to $\mathrm{T}$ and $\mathrm{R}$, and the coupling of $\mathrm{R}^{\prime}$ and $\mathrm{M}$ is ignored.We also limit the FRP is dominated by the bimolecular termination of $S$, whereas $\mathrm{CP}$ is dominated by the unimolecular termination of $\mathrm{S}^{\prime}$. More comlex systems including above couplings and the oxygen inhibition effects have been reported by Lin et al [26].

For comprehensive modeling we will use the so-called quasi-steady state assumption [15,18]. The life time of the singlet and triplet states of photosensitizer, the radicals ( $R, S$ and $\left.S^{\prime}\right)$, since they either decay or react with cellular matrix immediately after they are created. Thus, one may set $\mathrm{dT} / \mathrm{dt}=\mathrm{dR} / \mathrm{dt}=\mathrm{dS}^{\prime} / \mathrm{dt}=\mathrm{dR} " / \mathrm{dt}=0$, which give the quasi-steady-state solutions: $\mathrm{T}=\mathrm{bIg}[\mathrm{A}], \mathrm{S}^{\prime}=\mathrm{k}_{3}[\mathrm{C}] \mathrm{Tg}^{\prime}$, $S=\left(k_{3}[C]+k_{2}[B]\right) T g^{\prime \prime}$; with $g=1 /\left(k_{5}+k_{1}[A]+k_{2}[B]+k_{3}[C]+k M\right), g^{\prime}=1 /\left(k_{7}[B]+K^{\prime} M^{\prime}\right)$ and $g^{\prime \prime}=1 /\left(k^{\prime \prime} R+K M\right)$. Under this quasi-steady-state conditions, RGE =T/g- $\mathrm{k}_{1}[\mathrm{~A}] \mathrm{T}=\mathrm{bI}[\mathrm{A}]-\mathrm{k}_{1}[\mathrm{~A}] \mathrm{T}$, such that $\mathrm{d}[\mathrm{A}] / \mathrm{dt}=$ $(\mathrm{Ib}[\mathrm{A}]-\mathrm{RGE})=-\mathrm{k}_{1}[\mathrm{~A}] \mathrm{T}$. The approximated solution is given by $[\mathrm{A}]=\mathrm{A}_{0} \exp [-\mathrm{X}(\mathrm{t})]$, with $\mathrm{X}(\mathrm{t})$ is the time integral of $\mathrm{k}_{1} \mathrm{~T}(\mathrm{t})$. Therefore, when $\mathrm{k}_{1}=0$, or a very weak type-I coupling with type-II dominant, $\mathrm{d}[\mathrm{A}] / \mathrm{dt}=0$, which defines a perfect regeneration, or $[\mathrm{A}]=\mathrm{A}_{0}$ is a constant.

The steady state solution of Eq. (A7) is more complex, and is given by the solution of

$k^{\prime} R^{2}+G R-P=0$

where $G=k^{\prime \prime} S+k^{\prime} M$, and $P=\left(k_{1}[A]+k_{2}[B]\right) T$. Solving for $R$, we obtain

$R=\left(\frac{1}{2 k^{\prime}}\right)\left(-G+\sqrt{G^{2}+4 k^{\prime} H}\right)$

Analytic formulas of $\mathrm{R}$ is available under two special cases.

Case (i) for unimolecular termination dominant, or $G>>k^{\prime} P$, we obtain $R=P / G$, which is a linear increasing function of $\left(\mathrm{k}_{1}[\mathrm{~A}]+\mathrm{k}_{2}[\mathrm{~B}]\right) \mathrm{T} /\left(\mathrm{k}^{\prime \prime} \mathrm{S}+\mathrm{k}^{\prime} \mathrm{M}\right)$.

Case (ii) for bimolecular termination dominant, with $P>>G R$, we obtain, $R=\left[P / k^{\prime}\right]^{0.5}$. a nonlinear function of $\left[\left(\mathrm{k}_{1}[\mathrm{~A}]+\mathrm{k}_{2}[\mathrm{~B}]\right) \mathrm{T} / \mathrm{k}^{\prime}\right]^{0.5}$, a square root function.

For analytic formulas, we will consider a perfect catalytic cycle of the initiator, or when $G^{\prime \prime}=0$, $\mathrm{d}[\mathrm{A}] / \mathrm{dt}=0$, or $[\mathrm{A}]=\mathrm{A}_{0}$, is a constant. Also for the case that $\mathrm{g}=1 /(\mathrm{kM}), \mathrm{g}^{\prime}=1 /\left(\mathrm{K}^{\prime} \mathrm{M}^{\prime}\right)$ and $\mathrm{g}^{\prime \prime}=1 /(\mathrm{KM})$, for $[B]<<K^{\prime} M^{\prime}$, or $\left.[B]=0\right)$, such that $S^{\prime}=k_{3}[C] T^{\prime} /\left(k K^{\prime} M M^{\prime}\right), S=\left(k_{3}[C]+k_{2}[B]\right) T^{\prime} /\left(k_{K} M^{2}\right)$, with $T^{\prime}=b I A 0$. We first -order solution of $[B]$, using first order of $M=M_{0}$, and $M^{\prime}=M^{\prime} 0$; and $[C]=C_{0} \exp (-d t)$, with $d=k_{3} T^{\prime} /\left(k_{0}\right)$ to obtain $[B]=B_{0} \exp \left(-d^{\prime} t\right)$, with $d^{\prime}=k_{2} T^{\prime} /\left(k M_{0}\right)+k_{7} k_{3} T^{\prime}\left[C_{0} /\left(k M_{0} K^{\prime} M^{\prime}\right)\right] H(t)$, with $H(t)=[1-\exp (-d t)] / d$, which allows us to find R, and solve for the efficacy of FRP and CP from Eq. (A9) and (A10) as follows.

Time integral of $K^{\prime} S^{\prime}$ in Eq. (A10) leads to the solution fo $C P, M^{\prime}(t)=M_{0}{ }^{\prime}-K^{\prime} D\left(b I C_{0} A_{0}\right) H(t)$, with $H(t)=[1-\exp (-d t)] / d$, and $D=k_{7} k_{3}\left[C_{0} /\left(k M_{0} K^{\prime} M^{\prime}\right)\right.$. Therefore, the $C E$ of $C P$ is given by $C E^{\prime}=1-M / M_{0}$, or 
which has a transient state proportional to bIt, but a steady state given by $C^{\prime}=K^{\prime} D\left(b I C_{0} A_{0}\right) / d=$ $\mathrm{K}^{\prime} \mathrm{D}\left(\mathrm{C}_{0} \mathrm{~A}_{0}\right)\left[\mathrm{k}_{3} /(\mathrm{kM})\right]$, which is independent to the light intensity (I). (A13) is for the case that $\mathrm{g}^{\prime}=1 /\left(\mathrm{k}_{7}[\mathrm{~B}]+\mathrm{K}^{\prime} \mathrm{M}^{\prime}\right)=1 /\left(\mathrm{K}^{\prime} \mathrm{M}^{\prime}\right)$, when $[\mathrm{B}]=0$. The second-order solution of $\mathrm{S}^{\prime}$, in the presence of $[B]$, leads to a revised $C E^{\prime \prime}=\left[1-\mathrm{k}_{7} \mathrm{~B}_{0} /\left(\mathrm{K}^{\prime} \mathrm{M}^{\prime}\right)\right] C E^{\prime}$, with a reduction factor $\mathrm{F}^{\prime}=1-\mathrm{k}_{7} \mathrm{~B}_{0} /\left(\mathrm{K}^{\prime} \mathrm{Mo}^{\prime}\right)$. Above fomula is based on a perfect regeneration case. If we If we include the second-oder solution $[A]=A_{0} \exp \left[-X^{\prime} t\right]$, with $X^{\prime}$ is the time average of $X(t)$, the Eq. (A13) needs a revised $H(t)=\left[1-\exp \left(-d^{\prime} t\right)\right] / d^{\prime}$, with a revsied $\mathrm{d}^{\prime}=\mathrm{d}+\mathrm{X}^{\prime}$.

Similarly, the time integral of K'S' in Eq. (A9) leads to the solution for FRP given by

$\mathrm{CE}=\left(\mathrm{T}^{\prime} / \mathrm{M}_{0}\right) \mathrm{t}+\mathrm{KD} \mathrm{H}^{\prime}(\mathrm{t})+\mathrm{KD} \mathrm{D}^{\prime} \mathrm{H}^{\prime}(\mathrm{t})+\mathrm{P}^{\prime} \mathrm{H}^{\prime \prime}(\mathrm{t})$,

with $H^{\prime}(t)=\left[1-\exp \left(-d^{\prime} t\right)\right] / d^{\prime} ; D^{\prime}=k_{3}\left(C_{0} / Q\right) T^{\prime}, D^{\prime \prime}=k_{2}\left(B_{0} / Q\right)$, with $Q=\left(k K^{\prime} M^{\prime}\right)$. And $H^{\prime \prime}(t)$ depends on the solutions of $R$ given by Eq. (A12) as follows. For case (i) $R=\left[\left(k_{1}[A]+k_{2}[B]\right) T /\left(k^{\prime} M\right)\left[1-k^{\prime \prime} S /\left(k^{\prime} M_{0}\right)\right]\right.$, we obtain $H^{\prime \prime}(\mathrm{t})=\mathrm{Pt}+\mathrm{P}^{\prime} \mathrm{H}^{\prime \prime}(\mathrm{t})$, with $\mathrm{H}^{\prime \prime}=\left[1-\exp \left(\mathrm{d}^{\prime \prime} \mathrm{t}\right)\right] / \mathrm{d}$, with $\mathrm{P}=\mathrm{k}_{1}\left(\mathrm{~A}_{0} / \mathrm{M}_{0}\right) \mathrm{T}^{\prime}, \mathrm{P}^{\prime}=\mathrm{k}_{2}\left(\mathrm{~B}_{0} / \mathrm{M}_{0}\right) \mathrm{T}^{\prime}$, and d"= $\left[\mathrm{k}_{2} \mathrm{~T}^{\prime} /\left(\mathrm{kM}_{0}\right)+0.5 \mathrm{k}_{7} \mathrm{k}_{3} \mathrm{~T}^{\prime} \mathrm{t}\left[\mathrm{C}_{0} /\left(\mathrm{kM}_{0} \mathrm{~K}^{\prime} \mathrm{M}^{\prime}{ }_{0}\right)\right] \mathrm{t}\right.$. For case (ii) $\mathrm{k}^{\prime} \mathrm{R}=\left[\mathrm{k}^{\prime}\left(\mathrm{k}_{1}[\mathrm{~A}]+\mathrm{k}_{2}[\mathrm{~B}]\right) \mathrm{T}\right]^{0.5}$, we obtain $\mathrm{H}^{\prime \prime}(\mathrm{t})=$ $\mathrm{Vt}+\mathrm{V}^{\prime} \mathrm{H}^{\prime \prime \prime}(\mathrm{t})$, with $\mathrm{V}=\left(\mathrm{k}_{1}\left(\mathrm{~A}_{0} \mathrm{M}_{0}\right) \mathrm{T}^{\prime}\right)^{0.5}, \mathrm{~V}^{\prime}=\left(\mathrm{k}_{2}\left(\mathrm{~B}_{0} \mathrm{M}_{0}\right) \mathrm{T}^{\prime}\right)^{0.5}$. We note that all all the 4 terms in Eq. (A14) have transient state proportional to $T^{\prime} t$ (or bItAo). In comparison, the steady state of $H(t)=1 / d^{\prime}$, $H^{\prime \prime \prime}(t)=1 / d "$, which are independent to the light intensity (I). Similar to the CP case, we may include the second-oder solution $[A]=A_{0} \exp \left[-X^{\prime} t\right]$, with $X^{\prime}$ is the time average of $X(t)$, then Eq. (A16) needs a revision factor such that $T^{\prime}$ is revised to $T^{\prime \prime}=T^{\prime}\left[1-\exp \left(-X^{\prime} t\right)\right] / X^{\prime}$, which has a steady state solution independent to the light intensity (I).

\section{References}

1. Fouassier, J. P. \& Lalevée, J. Photoinitiators for Polymer Synthesis-Scope, Reactivity and Efficiency. Wiley-VCH Verlag GmbH \& Co. KGaA: Weinheim, Germany, 2012.

2. Yagci, Y., Jockusch, S. \& Turro, N.J. Photoinitiated polymerization: Advances, challenges and opportunities. Macromolecules 43, 6245-6260 (2010).

3. Ligon, S.C.; Liska, R.; Stampfl, J.; Gurr, M.; Mulhaupt, R. Polymers for 3D printing and customized additive manufacturing. Chem. Rev. 2017, 117, 10212-10290.

4. Takagishi, K.; Umezu, S. Development of the improving process for the 3D printed structure. Sci. Rep. 2017, 7, 39852.

5. Shusteff, M.; Browar, A.E.M.; Kelly, B.E.; Henriksson, J.; Weisgraber, T.H.; Panas, R.M.; Fang, N.X.; Spadaccini, C.M. One-step Volumetric Additive Manufacturing of Complex Polymer Structures. Sci. Adv. 2017, 3, 7.

6. Janusziewicz, R.; Tumbleston, J.R.; Quintanilla, A.L.; Mecham, S.J.; DeSimone, J.M. Layerless Fabrication with Continuous Liquid Interface Production. Proc. Natl. Acad. Sci. USA 2016, 113, 11703-11708.

7. Kelly, B.E.; Bhattacharya, I.; Heidari, H.; Shusteff, M.; Spadaccini, C.M.; Taylor, H.K. Volumetric Additive Manufacturing via Tomographic Reconstruction. Science 2019, 363, 1075-1079. 
8. de Beer, M.P.; van der Laan, H.L.; Cole, M.A.; Whelan, R.J.; Burns, M.A.; Scott, T.F. Rapid, Continuous Additive Manufacturing by Volumetric Polymerization Inhibition Patterning. Sci. Adv. 2019, 5, 8 .

9. van der Laan, H.L.; Burns, M.A.; Scott, T.F. Volumetric Photopolymerization Confinement through Dual-Wavelength Photoinitiation and Photoinhibition. ACS Macro Lett. 2019, 8, 899-904.

10. Childress, K.K., Kim, K., Glugla, D.J., Musgrave, C.B., Bowman, C.N. \& Stansbury, J.W. Independent control of singlet oxygen and radical generation via irradiation of a two-color photosensitive molecule. Macromolecules 52(13):4968-4978 (2019).

11. Scott, T.F.; Kowalski, B.A.; Sullivan, A.C.; Bowman, C.N.; McLeod, R.R. Two-Color Single-Photon Photoinitiation and Photoinhibition for Subdiffraction Photolithography. Science 2009, 324, 913-917.

12. Claudino,M.; Zhang,X.; Alim,M.D.;Podgoŕski,M.;Bowman, C. N. Mechanistic Kinetic Modeling of Thiol-Michael Addition Photopolymerizations via Photocaged "superbase" Generators: An Analytical Approach. Macromolecules 2016, 49 (21), 8061-8074.

13. Huang, S.; Sinha, J.; Podgorski, M.; Zhang, X.; Claudino M. Bowman, C.N. Mechanistic modeling of the Thiol-Michael addition polymerization kinetics: Structural effects of the Thiol and Vinyl monomers. Macromolecules 2018, 51, 5979-5988.

14. Chen, K.T.; Cheng, D.C.; Lin, J.T.; Liu, H.W. Thiol-Ene photopolymerization in thick polymers: kinetics and analytic formulas for the efficacy and crosslink depth. Polymers 11, 1640 (2019) doi:10.3390/polym11101640.

15. Kirschner, J.; Paillard, J.; Bouzrati-Zerell, M.; et al. Aryliodonium ylides as novel and efficient additives for radical chemistry: example in camphorquinone (CQ)/Amine based photoinitiating systems. Molecules 24(16), 2913 (2019); doi:10.3390/molecules24162913.

16. Wertheimer CM,; Elhardt C, ; Kaminsky, SM, et al. Enhancing rose Bengal photosensitized protein crosslinking in the cornea. Invest. Ophthalmol Vis Sci. 2019, 60, 1845-1852.

17. Schmitz $C_{\text {, }}$ Halbhuber A, Keil D,; Strehmel, B. NIR-sensitized photoinitiated radical polymerization and proton generation with cyanines and LED arrays. Prog. Org. Coat. 2016, 100, 32-46.

18. Bonardi $\mathrm{AH}$; Dumur F,; Grant TM,; et al. High performance near-Infrared (NIR) photoinitiating systems operating under low light intensity and in the presence of oxygen. Macromolecules, 2018, $51,1314-1324$.

19. Lin J.T.; Liu HW,; Chen KT,; Chiu YC, ; Cheng DC. Enhancing UV photopolymerization by a redlight pre-irradiation: kinetics and modeling strategies for reduced oxygen-inhibition. J Polymer Science, 2020, 58, 683-691, DOI:10.1002/pol.20190201.

20. Lin J.T.; Chen K.T.; Cheng D.C.; Liu, H,W. Dual-wavelength (UV and Blue) controlled photopolymerization confinement for 3D-printing: modeling and analysis of measurements. Polymers, 2019, 11, 1819. 
21. Lin, J.T.; Liu, H.W.; Chen, K.T.; Cheng, D..C. 3-wavelength (UV, blue, red) controlled photopolymerization: improved conversion and confinement in 3D-printing. IEEE Access, 2020, 8, 49353-49362.

22. Liu, S.; Chen, H.; Zhang, Y.; Sun, K.; et al. Monocomponent photoinitiators based on Benzophenone-carbazole structure for LED photoinitiating systems and application on 3D printing. Polymers, 2020, 12(6), 1394.

23. Abdallah, M..; Hijazi,A.; Lin, J.T.; Graff, B.; Dumur, F.; Lalevee, J. Coumarin Derivatives as Photoinitiators in Photo-Oxidation and Photo-Reduction Processes and a Kinetic Model for Simulations of the Associated Polymerization Profiles. App Polymer Material. 2020, 2,27692780.

24. Lin, J.T,; Cheng, D.C. Modeling the efficacy profiles of UV-light activated corneal collagen crosslinking. PloS One. 2017;12:e0175002.

25. Lin, J.T. Kinetics of Enhancement for Corneal Cross-linking: Proposed Model for a Two-initiator System. Ophthalmology Research, 2019, 10(3): 1-6; Article no.OR.49970 DOI: 10.9734/OR/2019/v10i330109

26. Lin, J.T,; Chen. K.T,; Cheng. D,C,; Liu. H,W. Modeling the efficacy of radical-mediated photopolymerization: the role of oxygen inhibition, viscosity and induction time. Front. Chem.2019, 7:760. doi: 10.3389/fchem.2019.00760. 